KfK 3062

November 1980

\title{
Polarized Targets in Nuclear and High Energy Physics
}

W. Heeringa

Institut für Kernphysik

Kernforschungszentrum Karlsruhe 



\section{KERNFORSCHUNGSZENTRUM KARLSRUHE}

Institut für Kernphysik

$\operatorname{KfK} \quad 3062$

Polarized Targets in Nuclear and High Energy Physics

Wabe Heeringa

Invited talk at the Fifth International symposium on Polarization Phenomena in Nuclear Physics, Santa Fe, U.S.A., August $11-15,1980$

Kernforschungszentrum Karlsruhe. GmbH, Karlsruhe 
Als Manuskript vervielfältigt

Für diesen Bericht behalten wir uns alle Rechte vor

Kernforschungszentrum Karlsruhe $\mathrm{GmbH}$

ISSN 0303-4003 


\section{Abstract}

Recent developments in polarization techniques for polarized targets in nuclear and high energy physics are reviewed. Both thermal equilibrium methods and dynamical methods are discussed.

POLARISIERTE TARGETS IN KERN- UND HOCHENERGIEPHYSIK

\section{Zusammenfassung}

Es wird ein Überblick gegeben über neue Entwicklungen zur Herstellung polarisierter Targets in Kern- und Hochenergiephysik. Sowohl die Methoden des thermischen Gleichgewichts als auch die dynamischen Polarisationsmethoden werden diskutiert. 
2. THERMAL EQUILIBRIUM POLARIZATION

3. POLARIZATION WITH NON-EQUILIBRIUM 7 METHODS

3.1 Dynamical polarization in solids using microwaves

3.2 spin refrigeration through sample rotation

3.3 Polarized targets from polarized beams 16 3.4 Optical pumping 
1. $I \quad N \quad T \quad R \quad O \quad D \quad U \quad C \quad T \quad I \quad O N$

The employment of polarized targets has proven to be a useful tool in nuclear and high energy physics during the last decade. Especially in high energy physics the total number and the different kinds of experiments carried out with polarized targets are growing rapidly. Quite a lot of effort has been invested in developing new target materials and new techniques. In this paper those developments are discussed that became applicable in the recent past or propably will do so in the near future. In the first section the polarization by means of thermal equilibrium methods is discussed. These methods have only been applied in the nuclear physics energy region up to now. The second part is dedicated to the non-equilibrium methods with the dynamical polarization technique being the most frequently applied one. Moreover, some other techniques belonging to this category such as rotating targets, optical pumping and targets produced with polarized beams, will be mentioned.

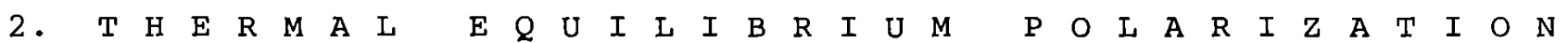

Magnetic moments can be oriented in space by putting them in a magnetic fieid. This also applies to nuclei with a magnetic moment, i.e. nuclei with spin $I \neq 0$. Nuclei are said to be oriented when the populations $\mathrm{p}(\mathrm{m})$ of the magnetic substates with quantumnumber $m$ are not all equal. The degree of orientation can be described by orientation parameters of increasing order $f_{1}, f_{2}, \ldots$. see e.g. the paper by ohlsen ${ }^{1}$. The first parameter is called the polarization and is expressed in the population numbers $p(m)$ as $f_{1}=\Sigma\{m p(m)\} / I$. The second parameter is called alignment parameter. 
When the magnetic moments are in thermal equilibrium with their environment, the magnetic energy $\mu \mathrm{H}$ should be comparable in size to the thermal energy $\mathrm{kT}$ in order to obtain a reasonable amount of polarization. To put things to scale, a nucleus with $\operatorname{spin} I=1$ and magnetic moment $\mu=1 \mu_{N}$ (nuclear magneton) obtains a polarization $\mathrm{f}_{1}=0.24$ in a field of $\mathrm{H}=10 \mathrm{~T}$ at a temperature $\mathrm{T}=0.01 \mathrm{~K}$. The techniques required to maintain such rather extreme values of $\mathrm{H}$ and $\mathrm{T}$ make polarized targets to be complicated and expensive.

The term brute force polarization is used when the magnetic field is supplied by an external magnet. On the other hand, in the hyperfine method the nucleus is polarized by an internal field created by unpaired electrons. Such internal fields are between 20 and $1000 \mathrm{~T}$, so they allow higher working temperatures. Generally only a modest external field is required to polarize the electrons completely, by which the internal fields are oriented parallel to each other. one prefers, of course, to employ targets containing only one nuclide. Several transition elements have unpaired electrons. These would therefore be the best candidates for a polarized mono-atomic target. Their nuclei must have a magnetic moment different from zero, of course. Another condition is, that they may not become anti-ferromagnetic at low temperatures. Four elements remain if one also requires that they are mono-isotopic, namely ${ }^{59} \mathrm{Co}^{2,3)},{ }^{159} \mathrm{~Tb}^{4)},{ }^{165}{ }_{\mathrm{HO}}^{5,6)}$ and ${ }^{169} \mathrm{Tm}$. Three more rare earths with more than one isotope satisfie the other requirements: Gd, Dy ${ }^{7}$ and Er.

Most of these elements have been used as polarized target in the past, mostly with polarized neutrons as projectiles. The references refer to some of these experiments and do not intend to be complete.

Another possibility for hyperfine polarization is to employ suitable compounds which have large internal fields. Some examples, that already have been employed are: Bi in Bimn ${ }^{8)}$, Au in AuFe and $U$ in $\mathrm{US}^{10)}$.

Instead of compounds one can also use salts in which the ion 
is paramagnetic. This has been done, for example, in the very first successfull experiment with polarized targets ${ }^{1}$ ), where ${ }^{55} \mathrm{Mn}$ nuclei were polarized in manganous ammonium sulfate. Another way in which many nuclides can be polarized is to introduce them into a ferromagnetic host lattice. The last two methods are frequently applied in nuclear spectroscopy where radioactive nuclei are oriented. They allow only small concentrations (a few percent or less) of the polarizable nuclei, however. This makes these materials unsuitable to be used as polarized targets in most cases.

Turning back to brute force polarization, the best nuclides to be polarized with this method are those with a large magnetic moment. Moreover, they should be metallic for two reasons. The first is to have sufficient heat conduction in the sample. The second is that only in metals the nuclear spin-lattice relaxation time is short enough to cool the nuclei in a reasonable time. some good candidates are $\left.\left.{ }^{7} \mathrm{Li},{ }^{27}{ }_{\mathrm{Al}}{ }^{12}\right),{ }^{45} \mathrm{Sc},{ }^{51} \mathrm{~V}^{13}\right),{ }^{55} \mathrm{Mn}$ etc.. The references refer again to some performed experiments. A more complete survey of favourable nuclei for the brute force method is given in a contribution to this conference ${ }^{14}$ ). A clear advantage of brute force polarization is that the targets can always be mono-atomic.

More and more nuclides can be polarized to a reasonable extent as one goes to higher fields and lower temperatures. Concerning the magnetic fields, one has gained now a lot of experience with the $\mathrm{Nb}_{3} \mathrm{Sn}$ multifilament superconductor technique.

For somewhat larger magnets with a bore between 5 and $10 \mathrm{~cm}$ a central field can be obtained around $10 \mathrm{~T}$ for a splitcoil and around 15 T for a solenoid.

From the low temperature front several interesting developments can be reported.

1. Attainment of $2 \mathrm{mK}$ by a dilution refrigerator running in the continuous mode and improved cooling powers at higher temperatures.

2. Better employment of the cooling power of enhanced nuclear magnetic systems. 
3. Attainment of temperatures in the low $\mu \mathrm{K}$ range by adiabatic demagnetization of $\mathrm{Cu}$ nuclei.

4. Polarization of solid ${ }^{3}$ He by Pomeranchuk cooling.

1. The performance of dilution refrigerators in the low mk region has been improved considerably in the last years. Fine powders are used in the heat exchangers and the mixing chamber of dilution refrigerators to increase the effective surface area, thus decreasing the thermal resistance (Kapitza resistance) between the helium and the metal. From the enthalpy balance between the concentrated and the dilute stream in a dilution refrigerator the following relation can be deduced (viscous heating is neglected):

$$
\mathrm{T}_{\mathrm{mc}}^{2}=1.1 \times 1 \mathrm{O}^{-2} \frac{\dot{\mathrm{Q}}}{\dot{\mathrm{n}}}+13 \frac{\dot{\mathrm{n}} \mathrm{R} K}{\sigma}
$$

Here $T_{m c}$ is the temperature of the mixing chamber, $\dot{Q}$ is the heat load on the mixing chamber, $\dot{n}$ is the circulation rate in mole/sec, $R_{K}$ the Kapitza resistivity and $\sigma$ the effective surface of the heat exchangers. The first term shows that large heat loads can be balanced by high circulation rates. It is clear from the second term that this only makes sense when the contact area is large enough. The lowest temperatures are achieved with $\dot{Q}$ as small as possible and $\sigma$ as large as possible.

It was believed up to about eight years ago that it would not be useful to employ powders smaller than $40 \mu$ because of the phononelectron resistance in the powder. Measurements of Radebaugh and siegwarth ${ }^{15}$ ) on $1.8 \mu$ powders showed this idea to be wrong. Experiments on still finer powders culminated in the work of Frossati and coworkers in Grenoble 16,17) three years ago. They employed silver powders of $700 \AA$ and later also of $400 \AA$ grain size and reached a temperature of $2 \mathrm{mK}$ in the continuous mode. Typical specific areas after sintering are $1.8 \mathrm{~m}^{2} / \mathrm{g}$ for $700 \AA$ powder and $2.2 \mathrm{~m}^{2} / \mathrm{g}$ for $400 \AA$ powder ${ }^{17}$ ). Fine silver powders are also used in commercially 
available dilution refrigerators now. The largest commercial machines can circulate up to 0.5 - $1 \mathrm{mmol} / \mathrm{sec}$. In fig. 1 a typical cooling power curve is shown for refrigerators circulating at $0.5 \mathrm{mmol} / \mathrm{sec}$.

2. Another method to attain temperatures in the low $\mathrm{mk}$ region is by demagnetization of enhanced nuclear magnetic systems ${ }^{18)}$. Without external field such systems have no free electrons, thus the electron cloud has no magnetic moment. However, an externally applied magnetic field induces an appreciable magnetic moment, which is proportional to the field. The magnetic moment creates a hyperfine field at the nucleus, which is stronger than the external field by the so called enhancement factor $(1+K)$. The situation can alternatively be described by an enhanced nuclear magnetic moment, which experiences only the external field. The enhanced moments have values in between the nuclear magneton and the Bohr magneton.

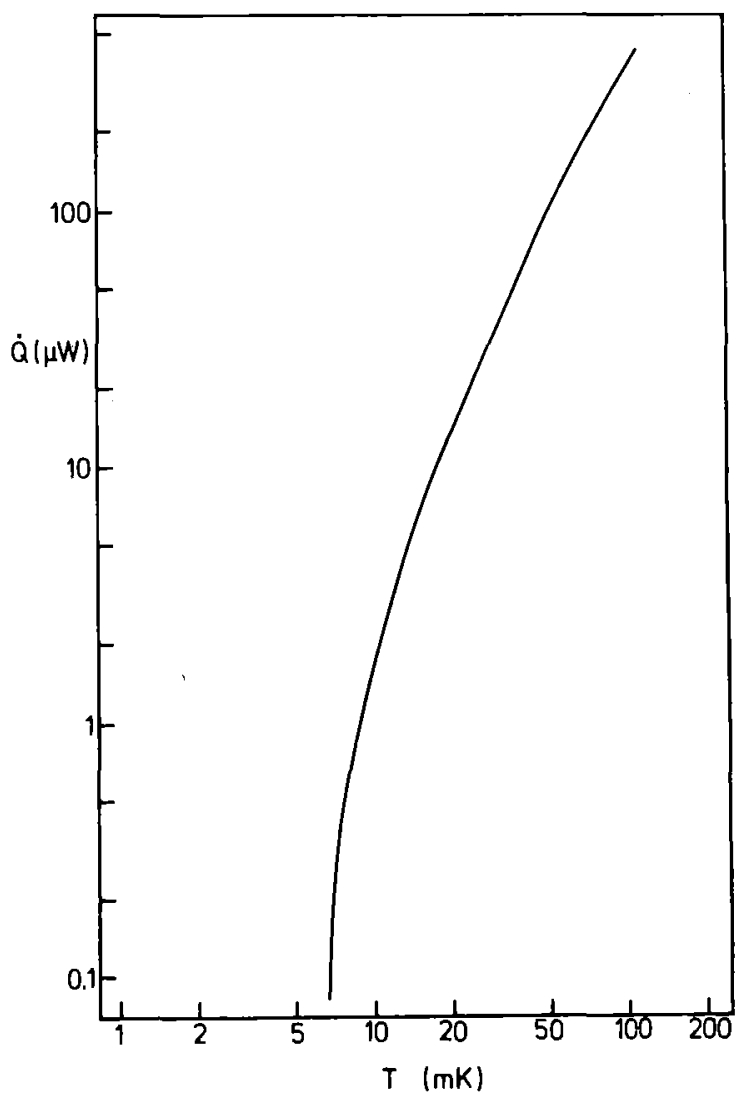

Fig.1: Typical cooling power curve of the largest dilution refrigerators, that can be commercially obtained nowadays, circulating at $0.5 \mathrm{mmol} / \mathrm{s}$. Suppliers are SHE, San Diego and OxfordInstruments, oxford 
The intermetallic compounds $\operatorname{PrCu}_{6}$ and PrNi 5 are suitable nuclear enhanced systems. They are used successfully now in many laboratories. Temperatures around $1 \mathrm{mk}$ can be reached with them. The improved cooling powers of dilution refrigerators allow a lower starting temperature for the demagnetization of the nuclear enhanced systems. The cooling power characteristic of fig.1 allows to reduce the entropy of a $1.5 \mathrm{~kg} \operatorname{PrCu}_{6}$ sample below 50\% of its full value within a few hours. This again yields a large cooling power of the $\mathrm{PrCu}_{6}$ at its lowest temperatures after demagnetization. A $1.5 \mathrm{~kg} \mathrm{PrCu}_{6}$ stage can keep another sample, with a heat load of $0.2 \mu \mathrm{w}$ on it, below $4 \mathrm{mK}$ for more than 40 hours ${ }^{19)}$. Such heat loads are typical for neutron experiments, thus the nuclear enhanced systems are a powerful tool in producing polarized targets. As far as I know they have not been employed as such yet.

3. Large samples of polarized Cu are produced by enhanced nuciear systems indeed, but they are employed to attain ultra low temperatures by nuclear demagnetization. The worldrecord in lattice temperature seems to be held at the moment by the Jülich group 20). After polarizing $600 \mathrm{~g}$ of $\mathrm{Cu}$ to $60 \%$ at $5 \mathrm{mK}$ and $8 \mathrm{~T}$, a lattice temperature of about $10 \mu \mathrm{K}$ was reached in this sample by demagnetization. During the demagnetization process the value of $\mathrm{H} / \mathrm{T}$ is constant, so the polarization remains unchanged.

4. Another way of reaching temperatures in the low mk range is by adiabatic compression of liquid ${ }^{3}$ He, also called Pomeranchuk cooling. A description of the principle can be found, e.g., in the book of Lounasmaa ${ }^{21}$ ). The method is based on the fact, that the entropy of solid ${ }^{3}$ He is larger than that of the liquid below $300 \mathrm{mK}$. This is opposite to the normal situation. Cooling occurs when liquid is forced to convert into solid by compression. Temperatures down to $1 \mathrm{mK}$ have been reached in this way ${ }^{21}$ ). When the compression takes place in a magnetic field, the solid ${ }^{3}$ He will become polarized. In 1973 Johnson et al. 22) 
obtained $47 \%$ average polarization in a liquid-solid mixture below $5 \mathrm{mK}$ in a field of $5.5 \mathrm{~T}$. Since then there have been some proposals to build such a target, but none has been realized yet, as far as I know.

3. $\mathrm{P}$ O $\begin{array}{llllllllllllllllllllll}B & R & I & U & M & M & E & T & H & O & D & S\end{array}$

\subsection{Dynamical polarization in solids using microwaves}

In these methods, an unequal population of the nuclear magnetic substates is attained by induced transitions between electron-nucleus spin states.

In the first years of experiments with polarized proton targets (the early sixties) the so called solid effect or Abragam-Jeffries effect was employed to produce polarization in LMN (Lanthanum Magnesium Nitrate). The mechanism will be recalled here briefly. The material is doped with a relatively small number of free electrons. These are polarized by placing the sample in a magnetic field ( $2.5 \mathrm{~T})$ at a sufficiently low temperature $(<1.5 \mathrm{~K})$. By irradiating the sample subsequently with microwaves, transitions are induced between electron-proton states. The frequency is chosen such, that an electron and a proton flip their spins simultaneously (fig.2 a $\rightarrow$ b). 


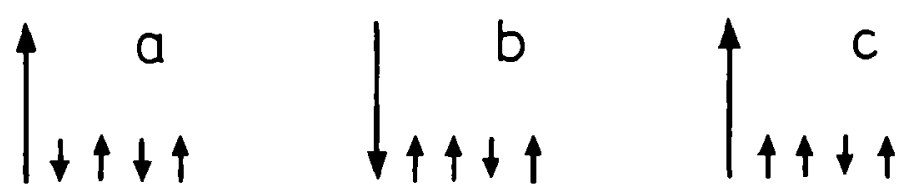

Fig.2: Schematic representation of nuclear polarization by the solid effect. Long arrows represent electron spins, short arrows nuclear spins. For further explanation, see text.

The electron is strongly coupled to the lattice, hence its spin flips back within a very short time (fig $2: b \rightarrow c)$, after which it can make a spin-flip with another nearby proton. The protons relax only very slowly to the lattice, hence their polarization is preserved.

Only a small number of free electrons is needed, because one electron can flip several protons and because the protons transfer the polarization to each other by means of mutual spin flips. The defects of LMN are its low hydrogen content and its low resistance to radiation damage. The low hydrogen content is especially a problem in high-energy physics, where one usually can not distinguish the scattering from free protons from that of the protons bound in nuclei. Thus here it is the ratio of free to bound protons $r$ that counts. In LMN $r=0.06$.

The advent of the organic materials (such as alcohols and diols) was a large step forward. First, the ratio $r$ is much higher (up to $r=0.24$ for butanol), and secondly, the radiation resistance is about a factor of 100 better. The physics behind the dynamical polarization in these substances is somewhat different from that in the solid effect. The procedure is the same, however, i.e., the sample is also doped with a small concentration of free electrons, kept at the same temperature, placed in a magnetic field of the same size and irradiated with 
microwaves of the same frequency range (about one hundred GHz). The difference is that in those materials electron spin pairs can exchange energies up to the nuclear zeeman energy. This causes the electron spin system (also called spin-spin interaction reservoir, because always two electron spins are involved) to be strongly coupled to the nuclei. It is possible to cool this reservoir by microwaves with frequencies lying on the slope of the electron spin resonance curve.

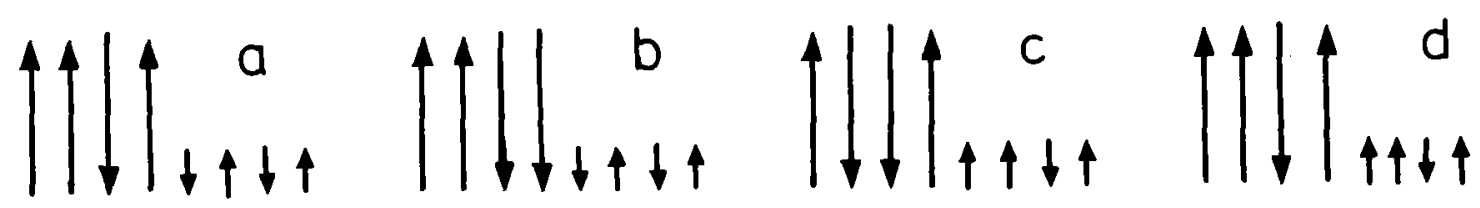

Fig.3: Schematical picture of dynamical orientation. An electron spin flips by absorption of microwaves ( $a+b)$. The electron spin-spin reservoir exchanges energy with the nuclei $(b \rightarrow c)$; the electron polarization is unchanged, the nuclear polarization increases. An electron relaxes back ( $c \rightarrow d)$.

Then the nuclei are also cooled due to their coupling to the spin-spin reservoir. The procedure is schematically indicated in fig.3. For a detailed treatment of the various ways of dynamical orientation I refer to the literature, e.g. the introduction given by Borghini ${ }^{3}$ ) and to the paper by Abragam and Goldman 24). The organic targets came into operation in the late sixties and are still the most popular materials in high energy physics.

I will now go through the most important developments made on target materials recently and then turn to the progress achieved in the experimental equipment.

Several difficulties had to be overcome in using organic targets:

i) one has to go below 1 k to achieve high polarization,

ii) most of them are liquid at room temperature, 
iii) suitable doping agents must be found to provide for the free paramagnetic centers.

The first point was solved by employing ${ }^{3}$ He refrigerators. Polarizations up to $90 \%$ can be obtained at the working temperature of about $0.5 \mathrm{~K}$. A favourite circumstance is that at $0.5 \mathrm{~K}$ the required microwave power is much smaller (by a factor of twenty or morel than at $1 \mathrm{k}$. The second problem has been tackled in two ways, namely, to search for polarizable materials that are solid at room temperature and to find methods by which the liquid ones can be handled at lower temperatures. Some solid materials have been found with fairly high polarizations, e.g. 1,8 octanediol 25 ) with $\mathrm{p}=60 \%$ and pinacol with $\mathrm{p}=70 \% 26$ ). They have not become common materials, however. The advantage of being solid obviously is outweighed by the disadvantage of lower overall polarization. The experimentalists have found ways to live with the liquid targets. They are stored under liquid nitrogen and can be loaded nowadays (also in dilution refrigerators) without warming up considerably, see e.g., ref.27).

One of the mostly practiced methods to obtain the paramagnetic centers is the use of $\mathrm{Cr}^{\mathrm{V}}$-complexes. They can be formed in the target material by chemical reduction of $\mathrm{cr}$ VI (e.g. $\mathrm{K}_{2} \mathrm{Cr}_{2} \mathrm{O}_{7}$ ). These complexes are unstable, however, hence it is difficult to arrive at the desired concentration and only little time is available for handling. The situation has been improved by the work of Krumpolc and Rotek ${ }^{28}$ ) about three years ago, who found stable $\mathrm{Cr}^{\mathrm{V}}$ complexes of tertiary hydroxy acids. These are widely used now. The target workshop of the Argonne high energy conference of october 1978 ${ }^{29}$ ) recommended as the best target material n-butanol-EHBA-Cr ${ }^{\mathrm{V}}$, EHBA being one of the stable complexes (2-ethyl-2-hydroxybutyric acid).

The polarization properties of target materials deteriorate severely after the bombardment of a certain amount of ionizing particles. It is known on the other hand, that paramagnetic centers are created by radiation damage. Therefore, one has investigated the possibility to polarize undoped samples that have 
been irradiated previously. In this case no interference would arise between the wanted and the unwanted paramagnetic impurities. Until 1978 only small polarizations were reported 29$)$. A breakthrough in this field has been achieved by Niinikoski and Rieubland ${ }^{30}$ ) at CERN last year. They irradiated solid $\mathrm{NH}_{3}$ to a total dose of $40 \mathrm{Mrad}$ by $580 \mathrm{MeV}$ protons and arrived at polarizations over 90\% afterwards. Ammonia is a very favourite material, because of the high value of $r=0.30$, the ratio of the free protons to the total number of protons. The authors estimate that the target would deteriorate only after an accumulated flux of more than $10^{17}$ minimum ionizing particles/cm ${ }^{2}$. This would bring an improvement of two orders of magnitude compared to the existing targets. Polarization results of the Yale-SLAC target group on irradiated targets will be presented at this conference 31 ).

Another interesting recent development has been reported by the group of Abragam at Saclay ${ }^{32}$. They polarized both the ${ }^{6} \mathrm{Li}$ and the deuteron nuclei in ${ }^{6}$ LiD to over 70\%. This is an interesting compound because not only the proton and the neutron of the deuterium are polarized, but also one neutron and one proton in the ${ }^{6}$ Li nucleus. The ${ }^{6}$ Li nucleus can be described either as ${ }^{4} \mathrm{He}+{ }^{2} \mathrm{H}$ or as ${ }^{3} \mathrm{He}+{ }^{3} \mathrm{H}$. Bot schemes yield the magnetic moment of ${ }^{6} \mathrm{Li}$ to an accuracy better than 4\%. And both schemes lead to the pairing off of two protons and two neutrons, leaving one proton and one neutron polarized. This brings us to the very high ratio $r=0.5$ for polarizable protons to total protons in ${ }^{6}$ LiD. The neutrons have the same ratio $r=0.5$, which surpasses even more all results obtained up to now. An inconvenience of the target is the long polarization build up time of one to two days.

Now I come to the developments in instrumental techniques. Generally, higher polarizations can be obtained if one goes to lower temperatures. However, the cooling machine must be able to carry away the heat generated by the microwave irradiation. The first targets were cooled to $1.0-1.5 \mathrm{~K}$ by a pumped ${ }^{4} \mathrm{He}^{-}$ bath. The next step was the ${ }^{3}$ He-cryostat. It can carry away 
large heat-loads down to $0.5 \mathrm{~K}$. A further step towards lower temperatures was made by the introduction of the dilution refrigerator. The first one to be used for dynamical polarization was built by Niinikoski at CERN in 197133). It has several advantages compared to the ${ }^{3}$ He-cryostat.

i) It can carry away large heat-inputs at still lower temperatures.

ii) The heat contact between the target and the coolant is better.

iii) The polarization can be frozen in by cooling rapidly to temperatures below $100 \mathrm{mK}$.

In the dilution refrigerator the target material (usually in the form of beads of about $1.5 \mathrm{~mm}$ diameter) is fully imbedaed in the liquid. The heat contact is governed by the kapitza resistance. In an evaporation ${ }^{3}$ He cryostat the heat resistance is the nucleate boiling resistance. Here the target is partially in contact with liquid and partially with gas. The result of the lower temperature and the better heat contact in the dilution refrigerator as compared to the ${ }^{3}$ He cryostat is given in fig.4 taken from ref. 29 .

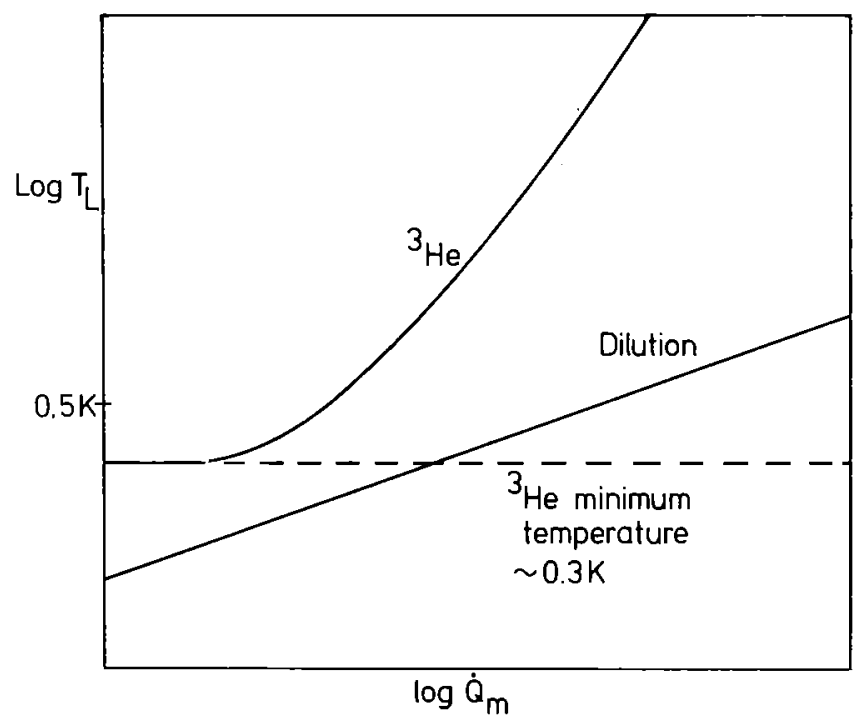

Fig. 4: Comparison of the calculated lattice temperature $T_{\text {of }}$ target materials cooled in a ${ }^{3} \mathrm{He}$ cryostat and in a dilution refrigerator as function of the heat load ¿े29). 
Here the lattice temperature $T_{L}$ of the target beads obtained in both cryostats is estimated as a function of the applied heat load $\dot{\dot{Q}}$. Over the whole range the dilution refrigerator gives the better results. It is the lattice temperature, of course, which finally determines the electron polarization and hence the nuclear polarization, that can be achieved. With ${ }^{3}$ He cryostats proton polarizations of about 90\% and deuteron polarizations of about $20 \%$ have been obtained. The advent of the dilution refrigerator has increased these numbers to $98 \%$ and $40 \%$ respectively. Thus, especially for deuteron targets, important progress has been made.

Very low temperatures can be reached with dilution refrigerators provided that the heat load is low enough. This feature is employed in the so called frozen spin targets. After the attainment of the desired amount of polarization, the microwave power is switched off and the target is cooled down quickly to below, say, $100 \mathrm{mk}$. In this temperature region the proton spin-lattice relaxation proceeds only very slowly, so that the polarization is maintained over many days without freshing up by microwave irradiation.

For example, the proton spin lattice relaxation time of 1,2-propanediol was measured as function of magnetic field and temperature by De Boer and Niinikoski ${ }^{34}$ ). Fig.5 taken from ref. 35 shows a smooth curve drawn through their experimental points measured at a field of $1 \mathrm{~T}$. 


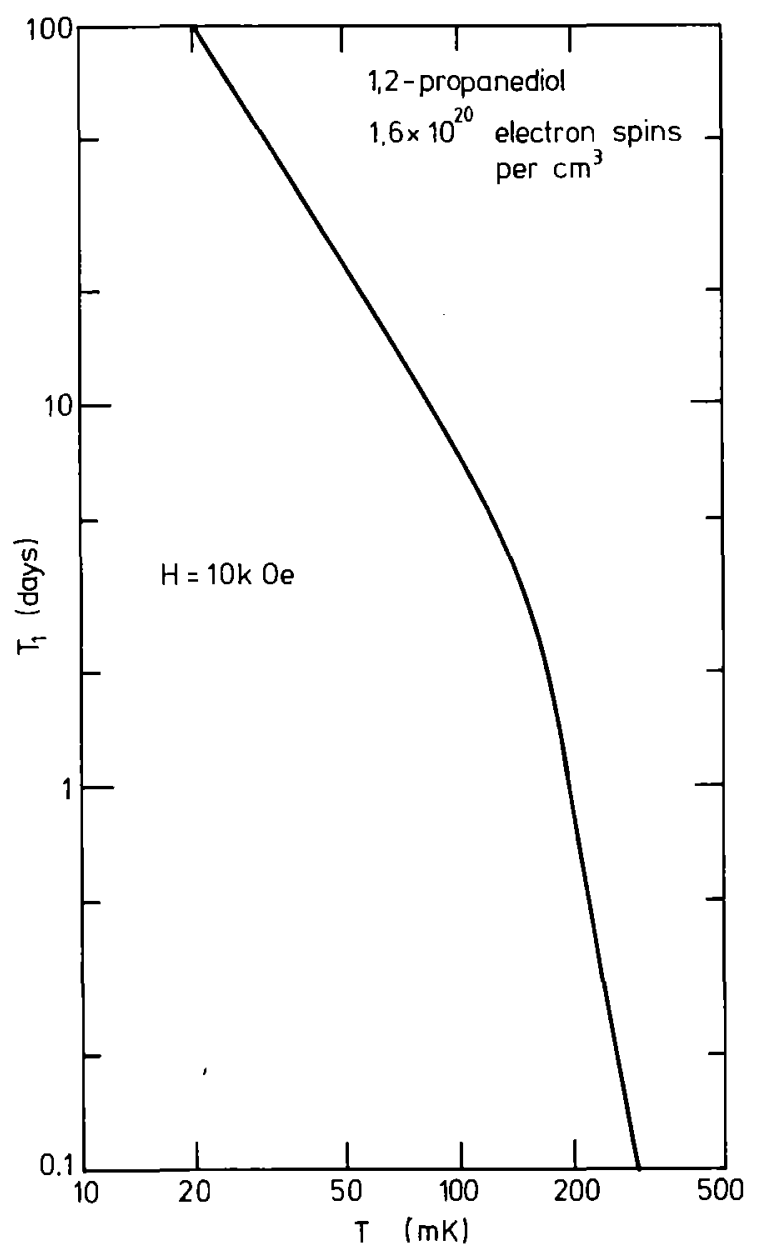

Fig. 5: Proton spin lattice relaxation time $T_{1}$ measured as function of $\mathrm{L}_{\text {the }}$ temperature 34 ).

At $100 \mathrm{mK}$ the relaxation time is already of the order of several days. The most important experimental improvement brought about by the frozen spin technique is given by the fact that the polarization in the holding mode can be maintained at a lower and far less homogeneous field than is required for the continuous irradiation mode. This allows for a larger solid angle of access to the target, which is very important to many types of experiments. For example, after polarization the target might be taken out of the polarizing magnet and moved into the field of the magnetic spectrometer that is used for the detection of the outcoming particles. Another advantage is the lower liquid helium consumption in the holding mode. The first frozen spin polarized target employing a dilution refrigerator, built at CERN by Niinikoski and coworkers ${ }^{27}$ ), was completed in 1974. 
Nowadays several high and medium energy laboratories have such a target in operation or are constructing one. A somewhat older review can be found in ref.36. A recent publication is, e.g., ref. 37 .

\section{2 Spin refrigeration through sample rotation}

Proton polarization is achieved in this technique by rotating a sample of yttrium ethyl sulfate (doped with a small amount of $\mathrm{Yb})$ in a nonuniform field of $1.1 \mathrm{~T}$ at a temperature of $1.25 \mathrm{~K}$. The polarization mechanism is based on the fact that, because of the internal crystallic fields, the Yb ion has a very anisotropic g-factor depending on the angle between the external field and the crystal c-axis. When these are parallel, one has $g_{\|}=3.33$, while in the perpendicular case $g_{\perp}$ is of the order of 0.003 , the size of the proton g-value, expressed in Bohr magnetons. During rotation of the crystal, the Yb ions mainly become polarized when the angle between the external field and the c-axis is $45^{\circ}$, where the electron spin-lattice relaxation is fastest and the g-factor is still large: $g\left(45^{\circ}\right)=2.35$. If the crystal is rotated at a rate larger than the Yb ion relaxation rate, then this polarization will be largely conserved at the time, that the c-axis gets perpendicular to the field. At this orientation a spin exchange may occur between the yb ions and nearby protons, which depolarizes the ions and polarizes the protons. During further rotation the Yb ions become polarized again, while the proton polarization is spread among the other protons by mutual spin flips. The attempts to build such a target started more than fifteen years ago 38,39 ). The results were reviewed by Jeffries ${ }^{40}$ at the Madison symposium ten years ago. At that time polarizations of $18 \%$ had been achieved by rotating crystals and 358 by rotating fields. Much higher polarizations were expected theoretically, however. The breakthrough came as it was discovered that much higher polarizations can be obtained by lowering the $\mathrm{Yb}$ concentration from $2 \%$ to $0.048^{41,42)}$. Polarizations up to $80 \%$ are achieved in the Uni- 
versity of Massachusetts spin refrigerator now ${ }^{43}$ ). The advantages are: low non-uniform field, temperature slightly above $1 \mathrm{~K}$ and no microwave system. The target material has a free to total proton ratio of $r=0.10$, in between LMN (0.06) and propanediol (o.19).

\section{3 Polarized targets from polarized beams}

Polarized targets produced with polarized beams have some interesting advantages compared to other targets. The polarization can be reversed rapidly, high polarizations may be obtained quite easily and clean experiments can be performed. The major drawback is, of course, the very small target thickness. On the other hand, very intense projectile beams can be tolerated. There are several possibilities: crossed-beam experiments, co-linear beams, storing the beam on a surface or storing it in a suitable chamber. The successfull storage of a polarized ${ }^{6}$ Li beam on a heated oxidized tungsten surface was reported at the zürich symposium by ulbricht and coworkers from the University of Hamburg ${ }^{44}$ ). The target thickness was $3 \times 10^{14}$ nuclei/cm ${ }^{2}$. The results of a scattering experiment performed with this target are presented at this conference ${ }^{45}$ ). Some more developments and proposals concerning these techniques are also presented at this conference $46-50)$.

\subsection{Optical pumping}

This method has been mainly used to produce polarized gaseous ${ }^{3}$ He of a pressure of some torr. The mechanism roughly proceeds as follows. Long-lived metastable atoms are produced in the gas by a discharge. The nuclei of these atoms become polarized by irradiating the gas with polarized light of a suitable frequency. By collisions with unpolarized ground state atoms this polarization can be transferred from the metastable to the ground state atoms. Several precise experiments have been done using such targets up to recently ${ }^{51}$ ). It appears to be possible to preserve the polarization while compressing the 
gas into the NTP range ${ }^{52}$. Attempts to polarize liquid ${ }^{3}$ He in this way have yielded only small polarizations ${ }^{3}$ ). Small polarizations in solids have also been reported ${ }^{54}$ ). The main activity in this field was about, ten years ago. No recent instrumental improvements have been reported in the last years as far as I know.

4. S S U

In the preceeding sections different kinds of polarized targets obtained by various methods have been discussed and some recent developments in polarizing techniques have been reviewed. In this summary some remarks will be added on their applicability to various experiments.

The brute force method requires the lowest temperatures $(<0.02 \mathrm{~K})$ and the highest magnetic fields (>5 T). The number of experiments employing this method has been relatively small, therefore, up to now. It may be expected that this number will increase in the near future, because of the improved cooling characteristics of dilution refrigerators. The brute force method is in principle applicable to all nuclides with spin $I \neq 0$. An important merit is the fact that the targets are mono-atomic. Tolerable heat inputs are below $1 \mu \mathrm{W}$, in favourable cases some $\mu w$. The allowed heat input is not only limited by the cooling power of the dilution refrigerator but also by the heat conduction in the sample itself. For this reason the method is mainly limited to the polarization of metal nuclei and to experiments with neutron beams. Brute force polarized targets have been employed in experiments to determine neutron resonance spins and in thermal neutron capture work to study the gamma decay of the compound nucleus. A systematic study of the spin-spin interaction of polarized MeV neutrons with several polarized nuclides is 
planned for the near Euture ${ }^{14}$ ).

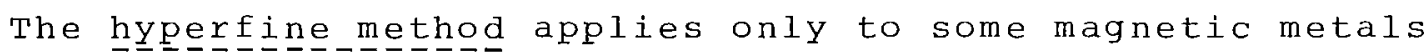
if pure elements are required. However, many transition elements can be polarized in simple compounds. The required temperatures are around $0.05-0.2 \mathrm{~K}$. These higher temperatures allow also experiments with charged particle beams, see, e.g., ref.55. Moreover, many neutron experiments of the types mentioned above have been performed with these targets ${ }^{8-10)}$.

Dýn larized proton and polarized deuteron targets, both for nuclear and for high energy physics experiments. The polarizations, especially of deuterons, have increased with the introduction of dilution refrigerators. New target materials will probably increase the merits of this method in the near future. The target dimensions can be increased enormously by building dilution refrigerators with higher circulation rates. At the moment a 1 m long, $5 \mathrm{~cm}$ diameter polarized proton target (butanol) is under construction at $\mathrm{CERN}^{56)}$.

The high circulation rates also allow very high heat loads on the target: $100 \mathrm{~mW}$ and more are possible. The problem of the bad heat conduction in the target materials (insulators) is overcome by employing small beads of $1 \mathrm{~mm}$ diameter that are immersed in the liquid. The method can basically been employed for all diamagnetic solids, provided that a suitable way of doping with free electrons can be found.

The important merit of the frozen fact that the target can be taken out of the polarizing field, which must be very homogeneous $\left(1: 10^{4}\right)$ and put into a weaker and far less homogeneous holding field. This allows a much better access to the target region.

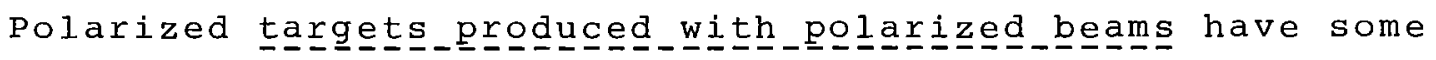
distinct advantages: no cryogenic equipment, clean targets, high polarizations can be obtained relatively easily and the polarization can be switched very fast. Only the effective target 
thickness is very small,but intense beams may be tolerated on the other hand. Several groups have started investigations in this new field.

In summarizing, one may say that many new developments in the polarized target technology are in progress, where especially the improvements in cryogenic technology play an important role. Thus it may be expected that new and better polarized targets will become available for experiments in the near future. 


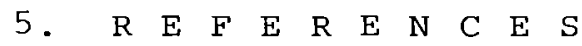

1) G.G.Ohlsen, Rep, Progr. Phys. 35 (1972) 717

2) T.R.Fisher et al., Nucl.Phys.A179 (1972) 241

3) W.Heeringa et al., Phys.Rev. C16 (1977) 1389

4) V.P.Alfimenkov et al., Sov.J.Nucl.Phys. 25 (1977) 495

5) G.Brunhart, H.Postma and V.L.Sailor, Phys.Rev. 137B (1965) 1484

6) T.R.Fisher, J.C.Healey and J.S.McCarthy, Nucl. Phys. A130 (1969) 609

7) G.Brunhart et al., Bull. Am. Phys. Soc. 15 (1970) 569

8) K.Nagamine, N.Nishida and T.Yamazaki, Proc. 2nd Int. Conf. on Polarized Targets, Ed.G.Shapiro, Berkeley, LBI 500 (1971) p.259

9) H.J.Ligthart and H.Postma, Z. Phys. 288A (1978) 179

10) M.S.Moore et al., Phys. Rev. C18 (1978) 1328

11) S.Bernstein et al., Phys.Rev.94 (1954) 1243

12) P.P.J.Delhey, ECN-Report 62, Petten, 1979

13) J.B.M. deHaas, ECN-Report 79, Petten, 1979

14) C.R.Gould et al., this conference

15) R.Radebaugh and J.D.Siegwarth, Proc. 13th Intern. Low Temp. Physics Conf., Boulder (1972) 401

16) G.Frossati et al., Proc. Hakone Int. Symp., Ed.T.Sugawara, Phys. Soc. of Japan 1978 , p. 205

17) G.Frossati, Journal de Physique C6 (1978) 1578

18) B. Bleaney, Physica 69 (1973) 317

19) J.A.Konter, Thesis, 1976, Leiden, unpublished 
20) R.M.Mueller et al., Phys.Lett. 75A (1980) 164

21) O.Lounasmaa, Experimental Principles and Methods below $1 \mathrm{~K}$, Academic Press, 1974

22 ) R.T.Johnson et al., J.Low Temp. Phys. 10 (1973) 35

23) M.Borghini, Proc. 2nd Int.Conf. on Polarized Targets, Ed. G.Shapiro, Berkeley, LBL 500 (1971) P.1

24) A.Abragam and M.Goldman, Rep. on Progr. Phys. 41 (1978) 395

25) P.Weymuth, Proc. 2nd Int. Conf. on Polarized Targets, Ed. G.Shapiro, Berkeley, LBL 500 (1971) p.301

26) H.Glättli, Proc. 2nd Int. Conf. on Polarized Targets, Ed. G.Shapiro, Berkeley, LBL 500 (1971) p.281

27) T.O.Niinikoski and F.Udo, Nucl. Instr. and Methods 134 (1976) 219

28) D.Hill, R.C.Miller, M.Krumpolc and J.Rőek, Nucl. Instr. and Methods 150 (1978) 331

29) G.R.Court et al., Proc. High Energy Symp. on Polarized Beams and Polarized Targets, Argonne, Ed. G.H.Thomas, AIP Conf. Proc. 51 (1979) 15

30) T.O.Niinikoski and J.M.Rieubland, Phys. Lett. 72A (1979) 141

31) M.L.Seely et al., this conference

32) V.Bouffard, Y.Roinel, P.Roubeau and A.Abragam, J. de Physique, to be published

33) T.O.Niinikoski, Nucl. Instr. and Methods 97 (1971) 95

34) W.de Boer and T.O.Niinikoski, Nucl. Instr. and Methods 114 (1974) 495 
35) T.O.Niinikoski, High Energy Physics with Polarized Beams and Targets, Argonne, Ed. M.L.Marshak, AIP Conf. Proc. 35 (1976)

36) A.S.L.Parsons, High Energy Physics with Polarized Beams and Targets, Argonne, Ed. M.L.Marshak, AIP Conf. Proc. 35 (1976) 508

37) S.Ishimoto et al., Nucl. Instr. and Methods 171 (1980) 269

38) K.H.Langley and C.D.Jeffries, Phys. Rev. Lett. 13 (1963) 808

39) J.R.MCCol1 and C.D.Jeffries, Phys. Rev. Lett. 16 (1966) 316

40) C.D.Jeffries, Proc. 3d Int. Symp. Madison, 1970, ed. H.H.Barschall and W.Haeberli, University Wisconsin Press, p.351

41) W.H.Potter and H.J.Stapleton, Phys.Rev. B5 (1972) 1729

$42)$ J.Button-Shafer, R.L.Lichti and W.H.Potter, Phys. Rev. Lett. $39(1977) 677$

43) J.Button-Shafer, Proc. High Energy Symp. on Polarized Beams and Targets, Argonne, Ed. G.H.Thomas, AIP Conf. Proc. 51 (1979) 41

44) J.Ulbricht et al., Proc. 4th Int. Symp. on Polarization Phen. in Nucl. Reactions, Zürich 1975, Ed. W. Grüebler and V.König Birkhäuser Verlag Basel, p.875

45) J.ulbricht et al., this conference

46) M.D.Barker et al., this conference

47) J.E.Clendenin and K.P.Schüler, this conference

48) C.A.Gagliardi et al., this conference

49) J.S.Dunham et al., this conference

50) D.E.Murnick, this conference 
51) G.Szaloky et al., Nucl. Phys. A303 (1978) 51

52) J.M.Daniels, Proc. 2nd Int. Conf. on Polarized Targets, Ed. G.Shapiro, Berkeley, 1971, LBL 500, p.77

53) H.H.McAdams, Phys. Rev. 170 (1968) 276

54) C.D.Jeffries, Proc. 2nd Int. Conf. on Polarized Targets, Ed. G.Shapiro, Berkeley, 1971, LBL 500, p.381

55) S.S.Hanna, Proc. 4th Int. Symp. on Pol. Phen. in Nucl. Reactions, Zürich 1975, p.407

56) M.Fidecaro, Proc. High Energy Symp. on Polarized Beams and Targets, Argonne, Ed. G.H.Thomas, AIP Conf. Proc. 51 (1979) 434 